\title{
Membership of 23 stars towards the bulge globular clusters NGC 6528 and NGC 6553*
}

\author{
P. Coelho ${ }^{1}$, B. Barbuy ${ }^{1}$, M.-N. Perrin ${ }^{2}$, T. Idiart $^{1}$, R. P. Schiavon ${ }^{3}$, S. Ortolani ${ }^{4}$, and E. Bica ${ }^{5}$ \\ 1 Universidade de São Paulo, Dept. de Astronomia, CP 3386, São Paulo 01060-970, Brazil \\ e-mail: pcoelho@usp.br; barbuy@iagusp.usp.br; thais@iagusp.usp.br \\ 2 Observatoire de Paris, 61 Av. de l'Observatoire, 75014 Paris, France \\ e-mail: Marie-Noel.Perrin@obspm.fr \\ 3 Observatório Nacional, rua General José Cristino 77, 20921-400 Rio de Janeiro, Brazil and \\ Present address: UCO/Lick Observatory, University of California, Santa Cruz, CA 95064, USA \\ e-mail: ripisc@ucolick.org \\ 4 Università di Padova, Dept. di Astronomia, Vicolo dell'Osservatorio 5, 35122 Padova, Italy \\ e-mail: ortolani@pd.astro.it \\ 5 Universidade Federal do Rio Grande do Sul, Dept. de Astronomia, CP 15051, Porto Alegre 91501-970, Brazil \\ e-mail: bica@if.ufrgs.br
}

Received 30 January 2001 / Accepted 8 June 2001

\begin{abstract}
Low resolution spectra of 23 stars towards the bulge globular clusters NGC 6528 and NGC 6553 are analysed. Radial velocities and atmospheric parameters are derived in order to check their membership in the clusters. Effective temperatures were obtained from photometric data for stars with $T_{\text {eff }}>3800 \mathrm{~K}$, whereas for cooler stars, they were derived from equivalent widths of $\mathrm{TiO}$ bands. Calibrations of $W(\mathrm{TiO})$ as a function of stellar parameters based on a grid of synthetic spectra are presented. Metallicities were derived from a comparison of the observed spectra to a grid of synthetic spectra. The sample comprises evolutionary stages from the Red Giant Branch to the Horizontal Branch, with parameters in the range $3200 \leq T_{\text {eff }} \leq 5000 \mathrm{~K}$ and $-0.5 \leq \log g \leq 2.4$. The mean metallicities obtained for NGC 6528 and NGC 6553 are $[\mathrm{Fe} / \mathrm{H}] \approx-0.5$ and -0.7 , in both cases with $[\mathrm{Mg} / \mathrm{Fe}]=+0.3$; assuming the same overabundance for the $\alpha$ elements $\mathrm{O}, \mathrm{Mg}, \mathrm{Si}, \mathrm{S}, \mathrm{Ca}$ and $\mathrm{Ti}$, this gives $\left[Z / Z_{\odot}\right]=-0.25$ and -0.45 . Membership verification by means of low resolution spectra is a crucial step in preparing targets for high resolution spectroscopy with $8 \mathrm{~m}$ class telescopes.
\end{abstract}

Key words. clusters: globular: NGC 6528, NGC 6553 - stars: abundances

\section{Introduction}

The study of the stellar populations in the Galactic bulge is very important to constrain possible models of galaxy formation. In particular, the determination of the metallicities and abundance ratios of bulge stars, either from the field or in clusters, provides key information to help decide among the possible scenarios for the history of chemical enrichment of the Galaxy.

There are few previous studies of radial velocities and metallicity estimations of bulge stars from low resolution spectra. In the analysis of 400 field bulge stars by Sadler et al. (1996), metallicities and $[\mathrm{Mg} / \mathrm{Fe}]$ values were estimated. Minniti (1995a,b) studied the membership of stars towards 7 bulge globular clusters.

\footnotetext{
Send offprint requests to: P. Coelho, e-mail: pcoelho@usp.br

* Observations collected at the European Southern Observatory - ESO, Chile.
}

The best studied among the bulge globular clusters are NGC $6528\left(\alpha_{2000}=18^{\mathrm{h}} 04^{\mathrm{m}} 49.6^{\mathrm{s}}, \delta_{2000}=\right.$ $\left.-30^{\circ} 03^{\prime} 20.8^{\prime \prime}\right)$ and NGC $6553\left(\alpha_{2000}=18^{\mathrm{h}} 09^{\mathrm{m}} 15.7^{\mathrm{s}}\right.$, $\left.\delta_{2000}=-25^{\circ} 54^{\prime} 27.9^{\prime \prime}\right)$. Ortolani et al. (1995) have shown that, besides being old, these clusters have luminosity functions which are very similar to that of Baade's Window, which indicates that they belong to the same stellar population.

NGC 6528 is located in the Baade Window, at a distance $d_{\odot}=7.83 \mathrm{kpc}$ from the Sun, and NGC 6553 is relatively close to the Sun, at a distance $d_{\odot}=5.1 \mathrm{kpc}$ (Barbuy et al. 1998). As they are both located in crowded fields, the measurement of radial velocities of individual stars is of crucial importance for the determination of their membership in the clusters.

Both clusters are known to be metal-rich. However, there is no consensus in the literature regarding their detailed metal abundances. Recently, Barbuy et al. (1999) analysed high resolution spectra of two giant stars of 
NGC 6553. An Iron abundance of $[\mathrm{Fe} / \mathrm{H}]=-0.55 \pm 0.2$ and abundance ratios $[\mathrm{Na} / \mathrm{Fe}] \approx[\mathrm{Al} / \mathrm{Fe}] \approx[\mathrm{Ti} / \mathrm{Fe}] \approx$ $+0.5,[\mathrm{Mg} / \mathrm{Fe}] \approx[\mathrm{Si} / \mathrm{Fe}] \approx[\mathrm{Ca} / \mathrm{Fe}] \approx+0.3$ were derived. These ratios imply an overall metallicity $\left[Z / Z_{\odot}\right] \approx-0.1$. Cohen et al. (1999), analysing high resolution spectra of five red horizontal branch stars, obtained a mean metallicity $[\mathrm{Fe} / \mathrm{H}]=-0.16$ and an excess of the $\alpha$-element calcium to iron of about 0.3 dex, which imply an overall metallicity $\left[Z / Z_{\odot}\right] \approx+0.1$. Metal abundances of these clusters are also discussed in Barbuy et al. (1999) and Barbuy (2000).

In view of the disagreement between previous determinations of $[\mathrm{Fe} / \mathrm{H}]$, it is important that abundance estimations be extended to a larger number of stars of both clusters. In this paper, we determine radial velocities, effective temperatures, gravities and estimations of metallicities $[\mathrm{Fe} / \mathrm{H}]$ based on low resolution spectra for 23 stars towards NGC 6553 and NGC 6528, and verify their membership in these clusters.

In Sect. 2 the observations are described. The radial velocities derived are presented in Sect. 3. In Sect. 4 the stellar parameters are derived, and synthetic spectra are compared to observations to estimate metallicities. Concluding remarks are given in Sect. 5 .

\section{Observations}

Low resolution spectra of individual stars of NGC 6528 and NGC 6553 were obtained in 1992 August and 1994 June, at the $1.5 \mathrm{~m}$ ESO telescope at ESO (La Silla). The Boller \& Chivens spectrograph was employed. In 1992 August the Thompson CCD \# 18 with $1024 \times 1024$ pixels, with a pixel size of $19 \mu \mathrm{m}$ was used. A resolution of $\Delta \lambda \sim 8 \AA$ and a spectral coverage of $\lambda \lambda 4800-8800 \AA$ were achieved. In 1994 June, the Ford Aerospace FA 2048 L, frontside illuminated, uncoated CCD detector (ESO \# 24) with $2048 \times 2048$ pixels and pixel size $15 \times 15 \mu \mathrm{m}$ was used. The grating \# 27 resulted in a spectral resolution $\Delta \lambda \sim$ $4 \AA$ and a spectral coverage in the range $\lambda \lambda 4800-7550 \AA$.

The log of observations is provided in Table 1 . The stars are identified according to the charts by Hartwick (1975) for NGC 6553 and van den Bergh \& Younger (1979) for NGC 6528. Spectra of a given star were co-added by weighting their $S / N$ ratios; the final $S / N$ are indicated in Table 1.

In Figs. 1 and 2 are shown the $V$ vs. $V-I$ ColourMagnitude Diagrams of NGC 6528 and NGC 6553 using data obtained with the Hubble Space Telescope (Ortolani et al. 1995) where the sample stars are identified.

\section{Radial velocities}

The radial velocities were determined by means of three methods, as explained below and reported in Table 2 . The observed radial velocities derived were transformed to heliocentric values using the observation dates given in Table 1.

(a) A Fourier cross-correlation was applied on the program spectra relative to selected template spectra.
Table 1. Log of observations.

\begin{tabular}{|c|c|c|c|c|c|}
\hline Star & $V$ & $V-I$ & Exp. (s) & Date & $S / N$ \\
\hline \multicolumn{6}{|c|}{ NGC 6528} \\
\hline I 1 & 16.10 & 1.93 & 5400 & 06.08.92 & 190 \\
\hline \multirow[t]{2}{*}{ I 2} & 15.73 & 2.59 & 1800 & 16.06 .94 & 25 \\
\hline & & & 5400 & 06.08 .92 & 75 \\
\hline I 5 & 15.37 & 2.22 & 2700 & 17.06.94 & 20 \\
\hline I 6 & 15.89 & 3.54 & 2700 & 17.06.94 & 40 \\
\hline II 8 & 15.71 & 2.19 & 2100 & 17.06.94 & 20 \\
\hline II 14 & 15.76 & 3.47 & 2700 & 17.06 .94 & 10 \\
\hline I 23 & 17.19 & 1.70 & 1800 & 16.06 .94 & 10 \\
\hline I 24 & 16.89 & 1.66 & 4500 & 17.06 .94 & 20 \\
\hline I 25 & 16.11 & 2.09 & 4500 & $16,17.06 .94$ & 40 \\
\hline \multirow[t]{2}{*}{ I 27} & 15.90 & 3.08 & 1800 & 16.06.94 & 20 \\
\hline & & & 5400 & 06.08 .92 & 100 \\
\hline I 36 & 16.41 & 1.98 & 2100 & 17.06 .94 & 20 \\
\hline II 39 & 15.88 & 2.30 & 2100 & 17.06 .94 & 30 \\
\hline I 40 & 15.93 & 2.08 & 2100 & 17.06.94 & 20 \\
\hline I 42 & 16.42 & 2.15 & 2100 & 17.06.94 & 30 \\
\hline II 70 & 15.85 & 2.36 & 1800 & 16.06 .94 & 20 \\
\hline \multicolumn{6}{|c|}{ NGC 6553} \\
\hline III 2 & 16.89 & 1.95 & 1800 & 16.06.94 & 20 \\
\hline \multirow[t]{2}{*}{ III 3} & 15.82 & 2.41 & 3600 & $14,16.06 .94$ & 25 \\
\hline & & & 5400 & 07.08 .92 & 100 \\
\hline III 17 & 15.36 & 3.01 & 1800 & 14.06.94 & 30 \\
\hline \multirow[t]{2}{*}{ II 51} & 15.48 & 2.54 & 1800 & 17.06 .94 & 10 \\
\hline & & & 5400 & 07.08 .92 & 80 \\
\hline \multirow[t]{2}{*}{ II 52} & 16.84 & 1.93 & 1800 & 17.06.94 & 40 \\
\hline & & & 5400 & 07.08 .92 & 230 \\
\hline II 85 & 15.52 & 2.51 & 3600 & $14,16.06 .94$ & 55 \\
\hline II 94 & 15.44 & 3.38 & 1800 & 17.06.94 & 20 \\
\hline II 95 & 15.73 & 2.64 & 1800 & 17.06 .94 & 35 \\
\hline
\end{tabular}

As templates, $12 \mathrm{G}, \mathrm{K}$ and $\mathrm{M}$ stars were selected from the Jacoby et al.'s (1984) library, which have approximately the same spectral resolution $(4.5 \AA)$ of the sample spectra. The templates adopted were the ones closest in spectral type to each of the program stars. The spectra of both sample and template stars were normalized and different regions in the spectra were defined in order to give highest peaks of cross-correlation for each considered template. The results obtained with this method are given in Col. 2 of Table 2. The rms of the values derived with each template spectrum is of the order of $15 \mathrm{~km} \mathrm{~s}^{-1}$. A systematic effect was identified for the coolest stars, since all of them appeared to show lower velocities when compared to the hotter stars of the same cluster and these values were not considered.

(b) Mean shifts between the observed wavelengths of identified absorption lines and laboratory wavelengths were measured (Col. 3 of Table 2). The rms of the values derived is of the order of $15 \mathrm{~km} \mathrm{~s}^{-1}$. 


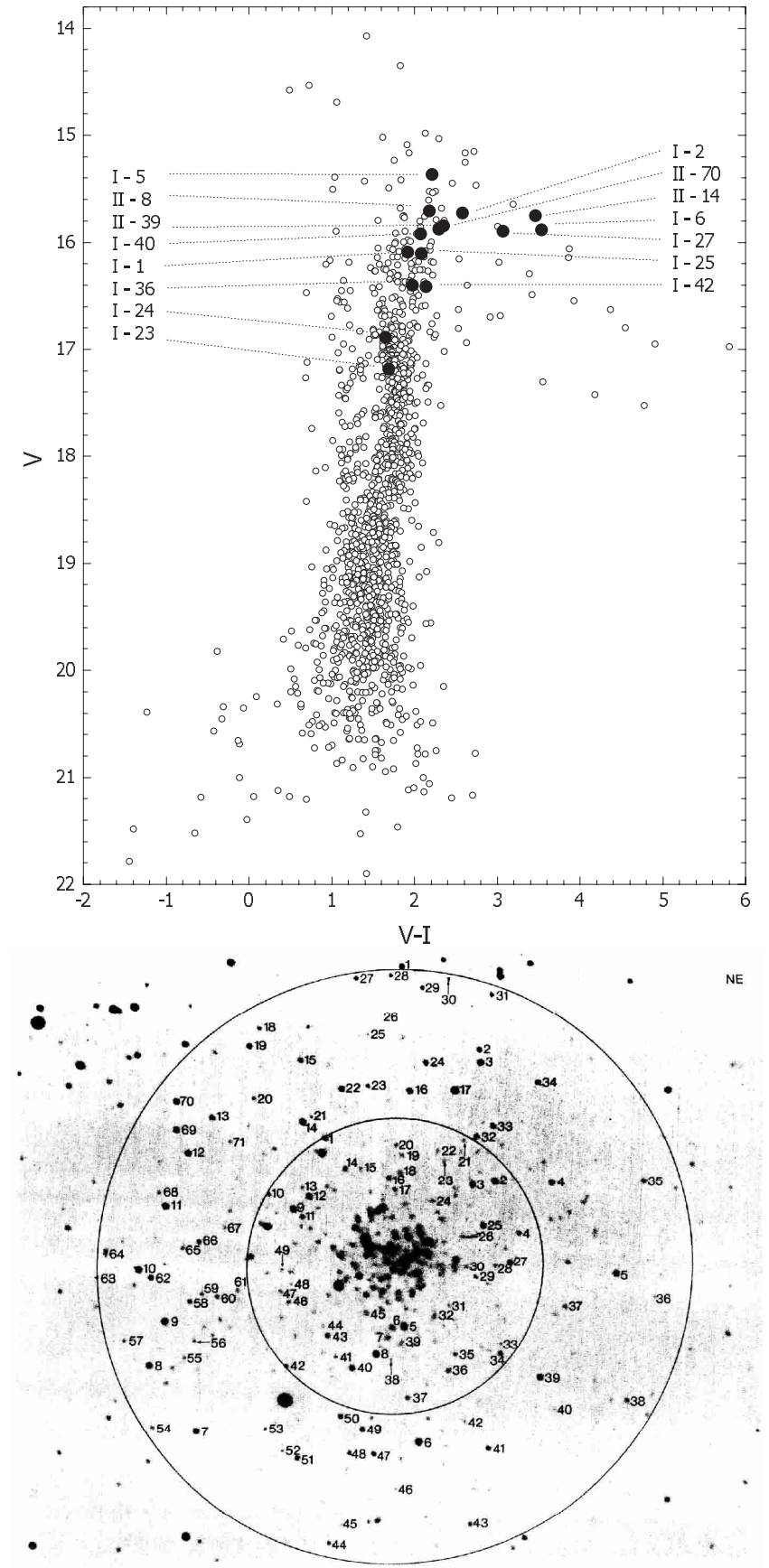

Fig. 1. Top: HST Colour-magnitude diagram of NGC 6528, where the observed stars are indicated as filled circles; Bottom: a map of the cluster scanned from van den Bergh \& Younger (1979).

(c) the code HALO (Cayrel et al. 1991) derives radial velocities by comparing the observed spectrum to a grid of synthetic spectra, using a cross-correlation technique. The grid of synthetic spectra available (Barbuy et al. 2001) does not contain stars cooler than $T_{\text {eff }}<4000 \mathrm{~K}$, and for this reason the errors should be higher for velocities of stars cooler than $T_{\text {eff }}<3700 \mathrm{~K}$ in which $\mathrm{TiO}$ bands are pronounced.

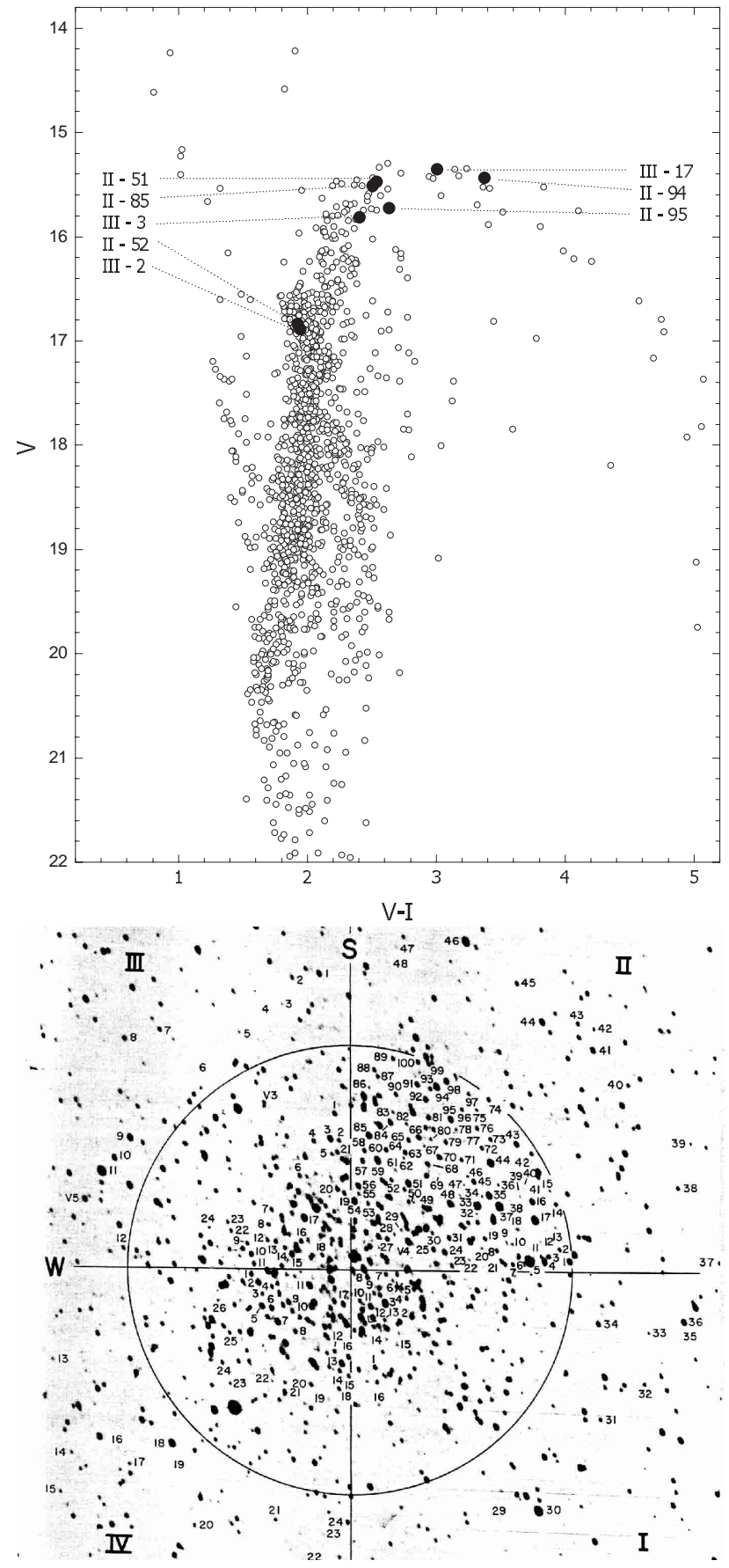

Fig. 2. Top: HST Colour-magnitude diagram of NGC 6553, where the observed stars are indicated as filled circles; Bottom: a map of the cluster scanned from Hartwick (1975).

Histograms of radial velocities of individual stars (coolest stars excluded) corresponding to each method were built. Gaussian curves were fitted to each histogram, from which the radial velocity corresponding to each method was derived, as reported in Table 3 together with values from the literature. An example of this procedure is presented in Fig. 3 for the cross-correlation technique using IRAF. The final radial velocities adopted for the clusters correspond to the mean of the values derived from the three methods. 
Table 2. Heliocentric radial velocities $v_{\mathrm{r}}\left(\mathrm{km} \mathrm{s}^{-1}\right)$ obtained with different methods: (a) cross-correlation relative to observed template spectra using IRAF; (b) mean shift in wavelength for a list of selected lines; (c) cross-correlation relative to synthetic spectra using the code HALO (see text).

\begin{tabular}{lccc}
\hline Star & method (a) & $\begin{array}{c}v_{\mathrm{r}}\left(\mathrm{km} \mathrm{s}^{-1}\right) \\
\text { method (b) }\end{array}$ & method (c) \\
\hline NGC 6528 & & & \\
I 1 & - & 262 & 174 \\
I 2 & 224 & 262 & 208 \\
I 5 & - & 261 & 224 \\
I 6 & 232 & 246 & 225 \\
II 8 & 289 & 283 & 271 \\
II 14 & - & 264 & 227 \\
I 23 & 221 & 251 & - \\
I 24 & 238 & 220 & 202 \\
I 25 & 265 & 257 & 231 \\
I 27 & - & 237 & 188 \\
I 36 & 244 & 249 & 229 \\
II 39 & 30 & 17 & 7 \\
I 40 & 246 & 236 & 244 \\
I 42 & 197 & 235 & 212 \\
II 70 & 230 & 263 & - \\
\hline
\end{tabular}

\section{NGC 6553}

\begin{tabular}{lccc} 
III 2 & -2 & -7 & -25 \\
III 3 & 5 & 17 & 18 \\
III 17 & 3 & 8 & -19 \\
II 51 & - & 60 & - \\
II 52 & -16 & -28 & -33 \\
II 85 & 35 & 56 & -4 \\
II 94 & -42 & -56 & -63 \\
II 95 & 11 & -12 & -9 \\
\hline
\end{tabular}

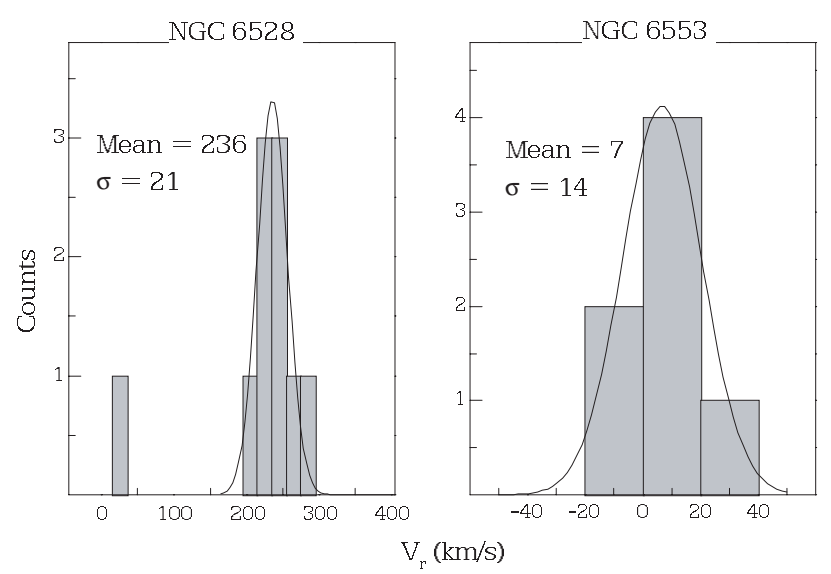

Fig. 3. Histograms of radial velocities obtained for the stars with the cross-correlation technique using IRAF, where the Gaussian fits are presented. The deviant point in the histogram of NGC 6528 is the star II 39, which is probably a non-member.

\section{Stellar parameters}

\subsection{Temperatures}

The effective temperatures were estimated from $B-V$, $V-I, V-K$ and $J-K$ colours, based on the colour
Table 3. Radial velocities of NGC 6528 and NGC 6553 given in the literature and values derived in the present work, for which the standard deviation values obtained with the Gaussian fits are indicated in parentheses.

\begin{tabular}{|c|c|c|}
\hline $\begin{array}{r}v_{\mathrm{r}} \\
\text { NGC } 6528\end{array}$ & $\begin{array}{l}\left.\mathrm{s}^{-1}\right) \\
\quad \text { NGC } 6553\end{array}$ & Reference \\
\hline 218 & 6.4 & 1,2 \\
\hline 164.8 & -24.5 & 3 \\
\hline 208 & 48 & 4 \\
\hline 212 & 8.4 & 5 \\
\hline 189 & - & 6 \\
\hline 160 & -5 & 7 \\
\hline 143 & -12 & 8 \\
\hline $236(21)$ & $7(14)$ & 9 \\
\hline $248(11)$ & $1(16)$ & 10 \\
\hline $217(9)$ & $-10(13)$ & 11 \\
\hline
\end{tabular}

References to Table: 1) Barbuy et al. (1999); 2) Barbuy (2000); 3) Harris (1996); 4) Minniti (1995) (mean values excluding nonmember stars); 5) Rutledge et al. (1997); 6) Armandroff \& Zinn (1988); 7) Zinn \& West (1984); 8) Zinn (1985); 9) present paper (cross-correlation method with IRAF); 10) present paper (mean wavelength shift with IRAF); 11) present paper (code HALO by a cross-correlation method).

vs. $T_{\text {eff }}$ calibrations by Bessell et al. (1998), which in turn are based on NMARCS models by Plez et al. (1992) and their grid extensions. These effective temperatures are listed in Table 5. $V$ and $I$ colours were obtained with the Hubble Space Telescope (Ortolani et al. 1995) and $J$ and $K$ colours were obtained with the detector IRAC2 at the $2.2 \mathrm{~m}$ telescope of ESO (Guarnieri et al. 1998).

For NGC 6528 the colour excesses adopted were $E(V-I)=0.68$ and $E(B-V)=0.52$ (Barbuy et al. 1998). For NGC $6553 E(V-I)=0.95$ and $E(B-V)=0.7$ were adopted (Guarnieri et al. 1998). The $V-K$ and $J-K$ colours were dereddened assuming $E(V-K) / E(B-V)=$ 2.744 and $E(J-K) / E(B-V)=0.527$ (Rieke \& Lebofsky 1985).

An independent method for the derivation of temperatures was based on calibrations of equivalent widths of $\mathrm{TiO}$ bands. The indices as defined in Table 4 were measured on the grid of synthetic spectra by Schiavon \& Barbuy (1999) in the range of parameters $3000 \leq T_{\text {eff }} \leq 5000 \mathrm{~K}$, $-0.5 \leq \log g \leq 2.5$ and $[\mathrm{Fe} / \mathrm{H}]=-0.3$. These indices are shown in Fig. 4 for a resolution of $\Delta \lambda=8 \AA$. Polynomial curves of the form $T_{\text {eff }}=f(W(\mathrm{TiO}))$ were derived and applied to the indices measured in the sample stars. The $\mathrm{TiO}$ indices are strongly sensitive to temperature for $T_{\text {eff }} \leq 3800 \mathrm{~K}$ as illustrated in Fig. 5. For $T_{\text {eff }} \geq 4000 \mathrm{~K}$ a degeneracy appears due to the fact that $\mathrm{TiO}$ bands are not present at these higher temperatures.

A more general polynomial of the form $\log W(\mathrm{TiO})=$ $\left(a+b \log T_{\text {eff }}+c \log g+d[\mathrm{Fe} / \mathrm{H}]+e\left(\log T_{\text {eff }}\right)^{2}+\right.$ $\left.f[\mathrm{Fe} / \mathrm{H}]^{2}+g[\mathrm{Fe} / \mathrm{H}] \log T_{\text {eff }}\right)$, valid in the range $2500 \leq$ $T_{\text {eff }} \leq 5000 \mathrm{~K},-0.5 \leq \log g \leq 2.5$ and $-0.5 \leq \mathrm{Fe} / \mathrm{H} \leq 0$ was derived. The coefficients of the formula above are 

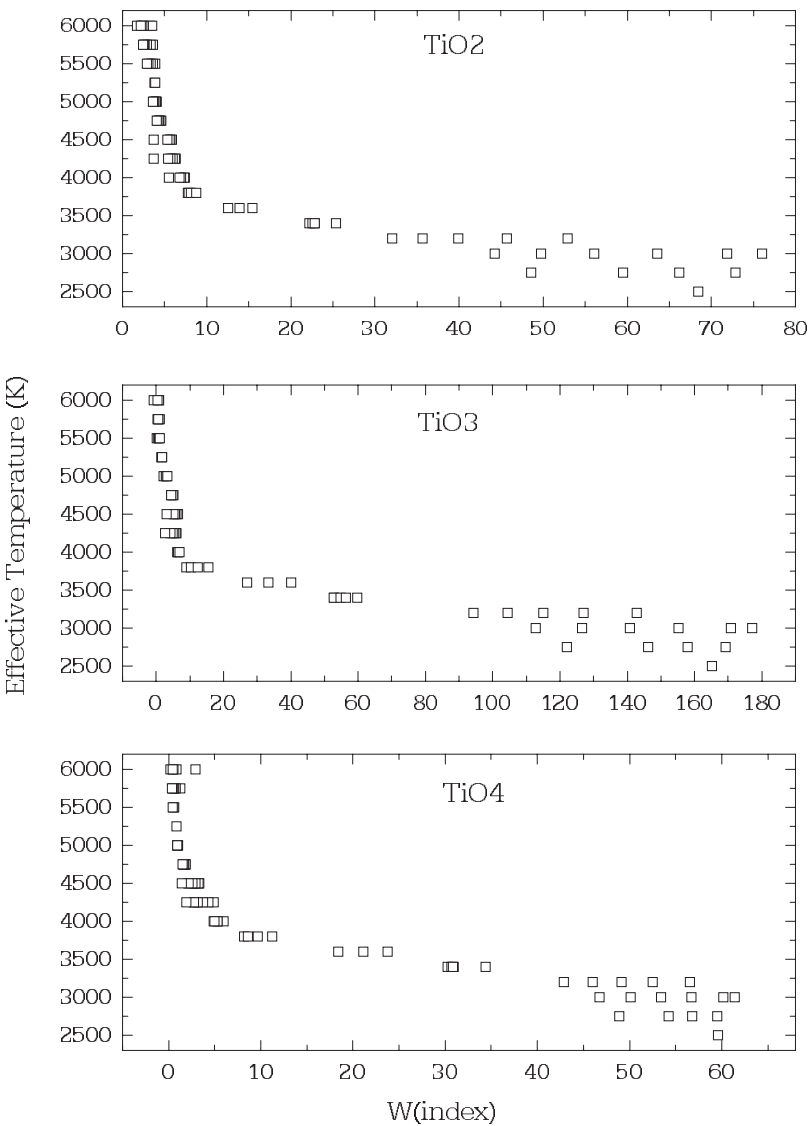

Fig. 4. $\mathrm{TiO}$ indices measured on the synthetic spectra as a function of effective temperatures. These measurements correspond to spectra convolved with $F W H M=8 \AA$.

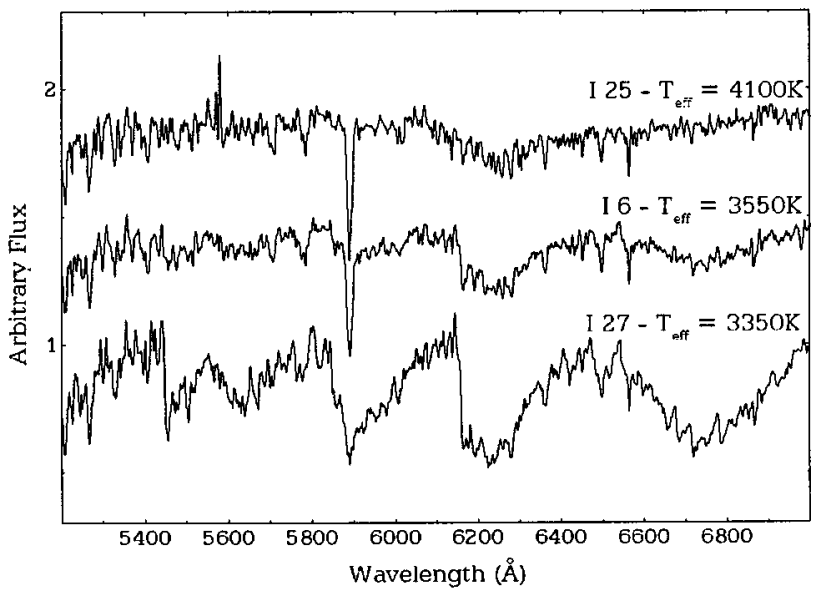

Fig. 5. Spectra of three individual stars of NGC 6528 with different temperatures. It is clear that the $T_{\text {eff }}=3350 \mathrm{~K}$ star has more pronounced $\mathrm{TiO}$ molecular bands with respect to the $T_{\text {eff }}=4100 \mathrm{~K}$ one.

shown in Table 6 for convolutions of $\Delta \lambda(F W H M)=4 \AA$ and $8 \AA$.

The temperatures obtained and final values adopted are reported in Table 5. Photometric temperatures were adopted for stars for which the $\mathrm{TiO}$ temperature
Table 4. TiO indices used in the derivation of effective temperatures. The definition of these indices is such as to avoid regions of strong telluric lines.

\begin{tabular}{cccc}
\hline Index & Blue continuum & Bandpass & Red continuum \\
\hline $\mathrm{TiO}_{2}$ & $6033.6-6050.6$ & $6300.0-6455.0$ & $6525.0-6538.0$ \\
$\mathrm{TiO}_{3}$ & $6525.0-6539.0$ & $6617.6-6860.0$ & $7036.0-7046.6$ \\
$\mathrm{TiO}_{4}$ & $7036.0-7046.6$ & $7053.0-7163.0$ & $7534.2-7546.8$ \\
\hline
\end{tabular}

Table 5. Effective temperatures based on calibrations of $B-V$, $V-I, V-K$ and $J-K$ colours (Cols. 2 to 5 ) and $\mathrm{TiO}$ indices (Cols. 6 to 8), and final values adopted. The discrepancy between the photometric and spectroscopic effective temperatures of II-51 might be explained by observations in different phases of the light curve of this probable red variable.

Effective Temperatures (K)

Star $V-I B-V J-K V-K \mathrm{TiO}_{2} \mathrm{TiO}_{3} \mathrm{TiO}_{4}$ Final

\begin{tabular}{lccllllll} 
NGC & 6528 & & & & & & & \\
I 1 & 4305 & 4449 & - & - & - & - & - & 4400 \\
I 2 & 3673 & 3883 & - & - & 3922 & 3698 & 3506 & 3700 \\
I 5 & - & - & - & - & 3468 & 3053 & - & 3250 \\
I 6 & 3451 & 3890 & - & - & 3612 & 3585 & 3405 & 3550 \\
II 8 & 3963 & 3992 & - & - & 3776 & 4049 & 3789 & 3950 \\
II 14 & - & - & - & - & 3468 & 3053 & - & 3250 \\
I 23 & 4783 & 4770 & - & - & - & - & - & 4800 \\
I 24 & 4883 & 4707 & - & - & - & - & - & 4800 \\
I 25 & 4074 & 4092 & - & - & - & - & - & 4100 \\
I 27 & - & - & - & - & 3673 & - & 3001 & 3350 \\
I 36 & 4218 & 4216 & - & - & - & - & - & 4200 \\
I 40 & 4084 & 4137 & - & - & - & - & - & 4100 \\
I 42 & 3999 & 4149 & - & - & - & - & - & 4050 \\
II 70 & 3810 & 3951 & - & - & - & - & - & 3900 \\
\hline NGC 6553 & & & & & & & \\
III 2 & 4828 & - & 4809 & 4559 & - & 3842 & 3776 & 3800 \\
III 3 & 4015 & - & 4221 & 3939 & 3782 & 3800 & 3775 & 3800 \\
III 17 & 3606 & - & 3966 & 3611 & 4052 & 3698 & 3503 & 3750 \\
II 51 & 3885 & - & 3966 & 3856 & 3281 & 3206 & - & 3250 \\
II 52 & - & - & - & - & 4241 & 3703 & 3752 & 3900 \\
II 85 & 3912 & - & 4140 & 3835 & - & - & - & 3950 \\
II 94 & 3506 & - & 3984 & 3432 & 4802 & 4408 & 3832 & 3650 \\
II 95 & - & - & - & - & - & - & - & 6500 \\
\hline
\end{tabular}

$T_{\mathrm{TiO}} \geq 3800 \mathrm{~K}$, whereas for stars with $T_{\mathrm{TiO}}<3800 \mathrm{~K}$ the mean of $\mathrm{TiO}$ temperatures were adopted.

\subsection{Gravities}

Gravities were derived using the classical relation $\log g_{*}=$ $4.44+4 \log T_{*} / T_{\odot}+0.4\left(M_{\mathrm{bol}}-M_{\mathrm{bol} \odot}\right)+\log M_{*} / M_{\odot}$, adopting $T_{\odot}=5770 \mathrm{~K}, M_{*}=0.8 M_{\odot}$ and $M_{\mathrm{bol} \odot}=4.74$ cf. Bessell et al. (1998). For deriving $M_{\text {bol* }}$ we used the distance modulus adopting a total extinction $A_{V}=2.43$ for NGC 6553 and $A_{V}=1.8$ for NGC 6528 (Barbuy et al. 1998). The bolometric magnitude corrections were taken from Bessell et al. (1998). 
Table 6. Coefficients of polynomial fits of $W(\mathrm{TiO})$ indices as a function of stellar parameters for $2500 \leq T_{\text {eff }} \leq 5000 \mathrm{~K}$, $-0.5 \leq \log g \leq 2.5$ and $-0.5 \leq[\mathrm{Fe} / \mathrm{H}] \leq 0$.

\begin{tabular}{lrrrrrr}
\hline Coefficient & \multicolumn{2}{c}{$\mathrm{TiO} 2$} & \multicolumn{2}{c}{$\mathrm{TiO} 3$} & \multicolumn{2}{c}{$\mathrm{TiO} 4$} \\
$\Delta \lambda$ & $4 \AA$ & $8 \AA$ & $4 \AA$ & $8 \AA$ & $4 \AA$ & $8 \AA$ \\
\hline$a(\operatorname{constant})$ & 81.49 & 81.89 & 55.70 & 56.51 & -175.11 & -158.38 \\
$b\left(\log \left(T_{\text {eff }}\right)\right)$ & -39.44 & -39.71 & -22.93 & -23.53 & 105.33 & 95.92 \\
$c(\log g)$ & -0.04 & -0.04 & -0.04 & -0.05 & -0.06 & -0.06 \\
$d([\mathrm{Fe} / \mathrm{H}])$ & 9.28 & 9.14 & 3.40 & 3.21 & -7.42 & -5.10 \\
$e\left(\log \left(T_{\text {eff }}\right)^{2}\right)$ & 4.75 & 4.79 & 2.17 & 2.27 & -15.66 & -14.34 \\
$f\left([\mathrm{Fe} / \mathrm{H}]^{2}\right)$ & -0.47 & -0.45 & -0.40 & -0.38 & -0.45 & -0.08 \\
$g([\mathrm{Fe} / \mathrm{H}] \times$ & & & & & & \\
$\left.\quad \log \left(T_{\text {eff }}\right)\right)$ & -2.56 & -2.51 & -0.87 & -0.82 & 2.16 & 1.54 \\
\hline$r^{2}$ & 0.92 & 0.92 & 0.90 & 0.90 & 0.97 & 0.96 \\
\hline
\end{tabular}

The resulting $M_{\mathrm{bol} *}$ and gravities are given in Table 7 . Taking into consideration the errors due to uncertainties in $T_{\text {eff }}$ and $M_{\text {bol }}$ the final error in $\log g$ is estimated to be of \pm 0.5 dex.

\subsection{Metallicities}

Spectrum synthesis calculations were used to fit the observed spectra. The calculations of synthetic spectra were carried out using the code described in Barbuy et al. (2000) where molecular lines of $\mathrm{MgH} \mathrm{A} \mathrm{A}^{2} \Pi-\mathrm{X}^{2} \Sigma, \mathrm{CH} \mathrm{A}^{2} \Delta-$ $\mathrm{X}^{2} \Pi, \mathrm{CN} \mathrm{A}^{2} \Pi-\mathrm{X}^{2} \Sigma, \mathrm{C}_{2}$ Swan $\mathrm{A}^{3} \Pi-\mathrm{X}^{3} \Pi$ and $\mathrm{TiO} \alpha \mathrm{C}^{3} \Delta-$ $\mathrm{X}^{3} \Delta, \gamma \mathrm{A}^{3} \Phi-\mathrm{X}^{3} \Delta$ and $\gamma^{\prime} \mathrm{B}^{3} \Pi-\mathrm{X}^{3} \Delta$ systems are taken into account.

For atomic lines the laboratory oscillator strengths by Fuhr et al. (1988), Martin et al. (1988), Wiese et al. (1969), and laboratory values compiled by McWilliam \& Rich (1994) were adopted whenever available, otherwise they were taken from fits to the solar spectrum (see discussion in Barbuy et al. 1999).

ATLAS9 and NMARCS models were employed. A grid of models using the ATLAS9 code (Kurúcz 1993) was created adopting a mixing length parameter $\alpha=0.5$ (see Barbuy et al. 2001). NMARCS photospheric models for giants by Plez et al. (1992) and their unpublished extended grids were employed (see more details in Schiavon \& Barbuy 1999).

The metallicities were obtained based on two methods, both using synthetic spectra:

(i) The observed spectra were compared to synthetic spectra in the range $\lambda \lambda$ 5000-7500 $\AA$. The metallicities were estimated by interpolating between synthetic spectra of $[\mathrm{Fe} / \mathrm{H}]=0.0,-0.3,-0.5$ and -0.6 , in all cases assuming $[\mathrm{Mg} / \mathrm{Fe}]=+0.3$, and the temperatures and gravities determined in Sects. 4.1 and 4.2.

(ii) Comparisons with a grid of synthetic spectra in the wavelength region $\lambda \lambda$ 4600-5600 $\AA$, using the differences method as described in Cayrel et al. (1991) and
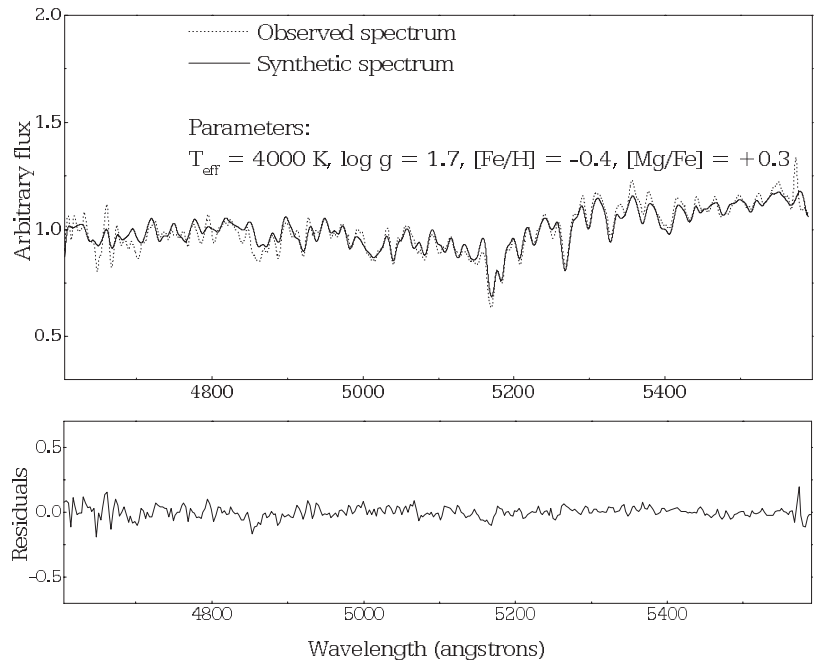

Fig. 6. Analysis of NGC 6528 I-1 employing the code HALO: Top: observed spectrum (dotted line) and synthetic spectrum (solid line) computed with $T_{\text {eff }}=4400 \mathrm{~K}, \log g=1.7,[\mathrm{Fe} / \mathrm{H}]=$ -0.4 and $[\mathrm{Mg} / \mathrm{Fe}]=+0.30(F W H M=8 \AA)$; Bottom: residual flux relative to the template synthetic spectrum.

Barbuy et al. (2001), are carried out. In this method, the observed spectrum is divided by a reference synthetic spectrum. The resulting signal can be expressed as a linear combination of variations in temperature, gravity and metallicity. In conjunction with the grid of synthetic spectra, it is possible to establish the differences in $T_{\text {eff }}, \log g$ and $[\mathrm{Fe} / \mathrm{H}]$ between the program star and the reference synthetic spectrum through a perturbation method. The grid covers the range $4000 \leq T_{\text {eff }} \leq 7000 \mathrm{~K}, 0.0 \leq \log g \leq$ $5.0,-3.0 \leq[\mathrm{Fe} / \mathrm{H}] \leq+0.3$, and $[\mathrm{Mg} / \mathrm{Fe}]=0.0$ and +0.4 . Figure 6 shows the fit to NGC 6528 I-1.

In Table 7 are listed the temperatures, gravities, $[\mathrm{Fe} / \mathrm{H}]$ and $[\mathrm{Mg} / \mathrm{Fe}]$ obtained with methods (i) and (ii). Note that method (ii) tends to give lower metallicities relative to method (i). This may be due to limitations of the grid of synthetic spectra, which is being extended to cover wider ranges of parameters.

The stars NGC 6528 I-5, NGC 6553 III-2 and II-95 are probable non-members, given that their atmospheric parameters are incompatible with their location in the Colour-Magnitude Diagrams of the clusters. The star II-51 appears to be too cool (Table 7) with respect to its CMD locus (Fig. 2). However, considering that it could be a red variable, this star is tentatively classified as a possible member.

Mean metallicities of $[\mathrm{Fe} / \mathrm{H}]=-0.5$ for NGC 6528 and $[\mathrm{Fe} / \mathrm{H}]=-0.7$ for NGC 6553 were estimated from Gaussian fits to the histograms of metallicities given in Table 7 (typical standard deviations are of $0.2 \mathrm{dex}$ ). These metallicities, together with the magnesium excess of $[\mathrm{Mg} / \mathrm{Fe}] \approx+0.3$, and assuming that all other $\alpha$ elements show an excess of +0.3 dex relative to iron, result in overall metallicities of $\left[Z / Z_{\odot}\right]=-0.25$ and -0.45 for NGC 6528 and NGC 6553 respectively. 
Table 7. Bolometric magnitudes, atmospheric parameters and method employed (see Sect. 4.3). $M_{\text {bol }}$ values were not reported for probable non-member stars.

\begin{tabular}{lcccccc}
\hline Star & $M_{\text {bol }}$ & $T_{\text {eff }}$ & $\log g$ & {$[\mathrm{Fe} / \mathrm{H}]$} & {$[\mathrm{Mg} / \mathrm{Fe}]$} & Method \\
\hline NGC 6528 & & & & & \\
I 1 & -0.8 & 4400 & 1.7 & -0.4 & 0.3 & $\mathrm{i}$ \\
& & 4400 & 1.7 & -0.4 & 0.3 & $\mathrm{ii}$ \\
I 2 & -2.0 & 3700 & 0.9 & -0.5 & 0.3 & $\mathrm{i}$ \\
I 5 & - & 3250 & 0.7 & -0.5 & 0.3 & $\mathrm{i}$ \\
I 6 & -3.0 & 3550 & 0.4 & -0.6 & 0.3 & $\mathrm{i}$ \\
II 8 & -1.5 & 4000 & 1.2 & -0.5 & 0.3 & $\mathrm{i}$ \\
& & 4000 & 0.5 & -0.6 & 0.3 & $\mathrm{ii}$ \\
II 14 & -3.1 & 3250 & 0.2 & -0.5 & 0.3 & $\mathrm{i}$ \\
I 23 & 0.6 & 4800 & 2.4 & 0 & 0.3 & $\mathrm{i}$ \\
& & 4800 & 2.4 & -0.4 & 0.2 & $\mathrm{ii}$ \\
I 24 & 0.4 & 4800 & 2.3 & 0 & 0.3 & $\mathrm{i}$ \\
& & 4800 & 2.3 & -0.3 & 0.1 & $\mathrm{ii}$ \\
I 25 & -1.0 & 4100 & 1.4 & -0.6 & 0.3 & $\mathrm{i}$ \\
& & 4100 & 1.4 & -1.1 & 0.2 & $\mathrm{ii}$ \\
I 27 & -2.5 & 3350 & 0.5 & -0.3 & 0.3 & $\mathrm{i}$ \\
I 36 & -0.6 & 4200 & 1.7 & -0.6 & 0.3 & $\mathrm{i}$ \\
& & 4250 & 1.0 & -0.8 & 0.4 & $\mathrm{ii}$ \\
I 40 & -1.2 & 4100 & 1.4 & -0.4 & 0.3 & $\mathrm{i}$ \\
& & 4100 & 1.4 & -0.7 & 0.3 & $\mathrm{ii}$ \\
I 42 & -0.8 & 4050 & 1.5 & -0.4 & 0.3 & $\mathrm{i}$ \\
& & 4050 & 1.5 & -1.2 & 0.2 & $\mathrm{ii}$ \\
II 70 & -1.6 & 3900 & 1.1 & -0.6 & 0.3 & $\mathrm{i}$ \\
\hline NGC 6553 & & & & & $\mathrm{i}$ \\
III 2 & - & 3800 & 2.0 & -0.7 & 0.3 & $\mathrm{i}$ \\
III 3 & -1.1 & 3800 & 1.3 & -0.7 & 0.3 & $\mathrm{i}$ \\
III 17 & -2.3 & 3750 & 0.8 & -0.7 & 0.3 & $\mathrm{i}$ \\
II 51 & - & 3250 & 0.8 & -0.4 & 0.3 & $\mathrm{i}$ \\
II 52 & 0.6 & 3900 & 2.0 & -0.6 & 0.3 & $\mathrm{i}$ \\
II 85 & -1.5 & 3950 & 1.2 & -0.6 & 0.3 & $\mathrm{i}$ \\
II 94 & -2.7 & 3650 & 0.6 & -1.1 & 0.3 & $\mathrm{i}$ \\
II 95 & - & 6500 & 2.7 & $-0.4:$ & - & $\mathrm{ii}$ \\
\hline & & & & & &
\end{tabular}

\section{Conclusions}

The study of individual stars in globular clusters along their evolutionary stages is of prime importance for an improved understanding of stellar evolution. Low resolution spectroscopy provides a means for the study of a large number of stars. In the present work we have measured radial velocities and estimated metallicities in 23 stars towards the globular clusters NGC 6528 and NGC 6553, which allows us to identify member stars. We also obtained their atmospheric properties to a first approximation. This is an important step before applying efforts to obtain high resolution spectroscopy with $8 \mathrm{~m}$ class telescopes. The method presented here is also of interest for last generation multi-object instruments such as VLT-VIMOS.

The stars were analysed by comparisons between their observed spectra and a grid of synthetic spectra. TiO equivalent widths were used to estimate effective temperatures of stars cooler than $T_{\text {eff }} \leq 3800 \mathrm{~K}$ and a calibration of equivalent widths of $\mathrm{TiO}$ bands as a function of atmospheric parameters is presented.

Mean values of heliocentric radial velocities of $v_{\mathrm{r}}=$ $234 \mathrm{~km} \mathrm{~s}^{-1}$ for NGC 6528 and $v_{\mathrm{r}}=-1 \mathrm{kms}^{-1}$ for NGC 6553 are derived.

Regarding membership, among the 23 stars observed we concluded that 4 of them are probable non-members. These are: NGC 6528 II-39, non-member due to a deviant radial velocity, and NGC 6528 I-5, NGC 6553 III-2 and II-95, non-members due to incompatibilities of atmospheric parameters vs. location in the Colour-Magnitude Diagrams.

NGC 6553 II-51 could be a non-member, or a red variable for which the spectrum was taken during a cool phase.

The basic stellar parameters derived show the interesting result that there is a trend for member giants of NGC 6528 to be more metal-poor than the two Horizontal Branch stars NGC 6528 I-23 and I-24, thus reproducing the discrepancy found between analysis of NGC 6553 giants by Barbuy et al. (1999) and Horizontal Branch stars by Cohen et al. (1999). Given the errors involved in the analysis of low resolution spectra, these results have to be checked with high resolution spectra, and further studies of this discrepancy will be possible only with a homogeneous analysis of stars ranging from the red giant branch to the HB, and also employing different sets of model atmospheres all along the evolutionary sequence.

In summary, we obtained for NGC 6528 and NGC 6553 metallicities of $[\mathrm{Fe} / \mathrm{H}]=-0.5 \pm 0.3$ and $[\mathrm{Fe} / \mathrm{H}]=$ $-0.7 \pm 0.3$. Using $[\mathrm{Mg} / \mathrm{Fe}] \approx+0.3$, and assuming that other $\alpha$ elements show the same excess of +0.3 dex relative to iron, the results are $\left[Z / Z_{\odot}\right]=-0.25$ and -0.45 for NGC 6528 and NGC 6553 respectively.

Acknowledgements. We are grateful to A. Milone for having carried out part of the observations. We acknowledge partial financial support from $\mathrm{CNPq}$ and Fapesp. P. Coelho and T. Idiart acknowledge respectively the Fapesp Master fellowship No. 98/07492-4, and Post-Doc fellowship No. 97/13083-7. RPS acknowledges support provided by the National Science Foundation through grant GF-1002-99 and from the Association of Universities for Research in Astronomy, Inc., under NSF cooperative agreement AST 96-1361.

\section{References}

Armandroff, T. E., \& Zinn, R. 1988, AJ, 96, 92

Barbuy, B., Bica, E., \& Ortolani, S. 1998, A\&A, 333, 117

Barbuy, B. 2000, in The Chemical Evolution of the Milky Way: Stars vs. Clusters, ed. F. Matteucci (Kluwer Acad. Pub.), in press

Barbuy, B., Renzini, A., Ortolani, S., Bica, E., \& Guarnieri, M. D. 1999, A\&A, 341, 539

Barbuy, B., Perrin, M.-N., Katz, D., et al. 2001, A\&A, submitted

Bessell, M. S., Castelli, F., \& Plez, B. 1998, A\&A, 337, 321

Carretta, E., \& Gratton, R. G. 1997, A\&AS, 121, 95

Cohen, J. G., Gratton, R. G., Behr, B. B., \& Carretta, E. 1999, ApJ, 523, 739 
Fuhr, J. R., Martin, G. A., \& Wiese, W. L. 1988, Atomic Transition Probabilities: Iron through Nickel, J. Phys. Chem. Ref. Data, 17(4)

Guarnieri, M. D., Ortolani, S., Montegriffo, P., et al. 1998, A\&A, 331, 70

Cayrel, R., Perrin, M. -N., Barbuy, B., \& Buser, R. 1991, A\&A, 247, 108

Harris, W. E. 1996, AJ, 112, 1487

Hartwick, F. D. A. 1975, PASP, 87, 77

Jacoby, G. H., Hunter, D. A., \& Christian, C. A. 1984, ApJ, 419, 592

Kurúcz, R. 1993, CD-ROM 18

Martin, G. A., Fuhr, J. R., \& Wiese, W. L. 1988, Atomic Transition Probabilities: Scandium through Manganese, J. Phys. Chem. Ref. Data, 17(3)

McWilliam, A., \& Rich, R. M. 1994, ApJS, 91, 749 (MR)
Minniti, D. 1995a, A\&A, 303, 468

Minniti, D. 1995b, A\&AS, 113, 299

Schiavon, R. P., \& Barbuy, B. 1999, ApJ, 510, 934

Ortolani, S., Renzini, A., Gilmozzi, R., et al. 1995, Nature, 377, 701

Plez, B., Brett, J. M., \& Nordlund, Å 1992, A\&A, 256, 551

Rieke, G. H., \& Lebofsky, M. J. 1985, ApJ, 288, 618

Rutledge, G. A., Hesser, J. E., Stetson, P. B., et al. 1997, PASP, 109,883

Sadler, E., Rich, R. M., \& Terndrup, D. M. 1996, AJ, 112, 171 van den Bergh, S., \& Younger, F. 1979, AJ, 84, 1305

Wiese, W. L., Martin, G. A., \& Fuhr, J. R. 1969, Atomic Transition Probabilities: Sodium through Calcium, NSRDS-NBS 22

Zinn, R. 1985, ApJ, 293, 424

Zinn, R., \& West, M. J. 1984, ApJS, 55, 45 